(Aus der Kgl. Universitäts-Augenklinik zu Halle a. S.

[Direktor: Prof. Dr. F. Schieck].)

\title{
Klinische Beobachtungen mit der Nernstspaltlampe und dem Hornhautmikroskop.
}

13. Mitteilung.

Weitere Erfahrungen über die an der Nernstspaltlampe zu beobachtende glaukomatöse Pigmentverstäubung im Irisstroma nebst Bemerkungen über deren Beziehungen zu Störungen des Sympathicus, speziell bei der Heterochromie.

Von

Privatdozent Dr. med. Leonhard Koeppe, Assistenzarzt.

Mit Tafel II.

\section{Inhalt.}

A. Einleitung.

B. 1. Weitere Erfahrungen über die an der Nernstspaltlampe zu beobachtende glaukomatöse Pigmentverstäubung im Irisstroma

a) bei klinisch sicheren Primärglaukomen;

b) bei Präglaukomen;

c) bei einigen neuen „Bindeglied-“ oder „Brückenfällen“.

2. Die Beziehungen der glaukomatösen Pigmentverstäubung im Irisstroma

a) zu Störungen des Sympathicus im allgemeinen;

b) zu der Heterochromie im besonderen.

C. Zusammenfassung und Schlußbemerkungen.

D. Literatur.

Seit meiner Mitteilung auf dem Heidelberger Kongresse im Jahre 1916 und der Drucklegung meiner ausführlichen Publikation in von Graefes Archiv, Band 92, Heft 3 über die Rolle und die Bedeutung des Irispigments bei der Entstehung des primären Glaukoms sowie die Glaukomfrühdiagnose mit der Gullstrandschen Nernstspaltlampe haben wir an dem großen Materiale unserer Klinik eine längere Reihe neuer Fälle verzeichnen können, die teils als klinische und manifeste Giaukome die in den oben erwähn̈ten Veröffentlichungen beschriebenen Pigmentverhältnisse in den verschiedensten Variationen darboten, teils ohne klinische Nachweismöglichkeit als Präglaukome zu registrieren waren. Wir möchten daher in der heutigen Mitteilung noch einmal auf 
das interessante und merkwürdige Kapitel der am lebenden Auge zu beobachtenden mikroskopischen Pigmentverhältnisse bei ausgesprochenen Glaukomen sowohl als bei Präglaukomen zurückkommen und im Anschlusse daran über einige neue Fälle derjenigen Art berichten, welche wir in Mitteilung 3 als sog. „Bindeglied-“ oder „Brückenfälle“ kennengelernt haben und die uns den Beweis zu geben scheinen, daß die an der Spaltlampe erkennbaren Pigmentveränderungen tatsächlich den klinischen Erscheinungen des Primärglaukoms vorausgehen und für die Frühdiagnose und evtl. auch für die Genese des Glaukoms von Bedeutung sein können.

Von besonderem. Interesse ist der Umstand, daß die in der genannten Mitteilung 3 anhangsweise aufgerollte Frage der Beziehungen des Halssympathicus einerseits und der Heterochromie andererseits zum Glaukom dadurch für uns wichtig wurde, als es uns gelang, bei 3 Fällen von Halssympathicusalteration das Bestehen von je einem Präglaukom und einem klinisch manifesten Glaukom, bei 3 Patienten mit Heterochromie in 2 Fällen ein Präglaukom und in einem Falle ein klinisches Glaukom zu beobachten.

Beginnen wir unsere Betrachtungen mit denjenigen vielgestaltigen Bildern der Pigmentverschiebung, die wir seit Drucklegung der oben angeführten Arbeiten wieder in zahlreichen einschlägigen Fällen zu verzeichnen Gelegenheit hatten, so können wir uns bezüglich der Kasuistik insofern kurz fassen, als wir, um Wiederholungen zu vermeiden, zusammenfassend über die seitdem an unserer Klinik beobachteten sicheren Fälle von Primärglaukom betreffs des Auftretens und der Erscheinungsformen ihrer Pigmentveränderungen berichten wollen.

Aus technischen Gründen sei vorher noch kurz von einem Kunstgriffe berichtet, der sich uns bei der Beobachtung der glaukomatösen Pigmentverhältnisse an der Gullstrandschen Nernstspaltlampe bei allen einschlägigen Fällen regelmäßig gut bewährte. Wir setzen nämlich zur besseren Sichtbarmachung des dunkelbraunen Irispigmentes auf ein kleines Reiterchen unmittelbar vor dem Spalt der Nernstspaltlampe eine Blauscheibe von bestimmter Tönung, etwa die Stärke A der Blaugläser von Ramin und Balthasar in Rathenow. Durch diese Blauscheibe sehen wir dann nämlich das dunkelbraune Pigment tiefschwarz gefärbt und sich um vieles besser von dem übrigen Irisgewebe sowohl als auch dem viel blasser erscheinenden Stromapigmente unterscheiden. Namentlich bei stromatisch stärker pigmentierten Regenbogenhäuten bedeutet diese Vorschaltung der Blauscheibe eine wesentliche Erleichterung bei der hier nötigen hauptsächlichen Durchforschung der Krypten und Lacunen, woselbst, wie wir seinerzeit darlegen konnten, bei diesen Fällen fast ausschließlich die Fest- 
stellung der Pigmentversehiebung gelingt. Nur bei wenigen Fällen blieb auch mit Blauscheibe der Nachweis glankomatöser Pigmentveränderungen unmöglich. Das Stromapigment selbst erscheint mit Blauscheibe niemals so tiefdunkel wie das epitheliale Pigment, die Blauscheibe hebt hier den Unterschied noch um vieles stärker hervor als bei offener Beleuchtung, ganz gleich, ob man in direktem oder indirektem Lichte untersucht.

Bemerken wollen wir noch, daß sich auch die Anwendung des schon öfters erwähnten schwarz mattierten Gehäuses auf dem Spaltarm für die Glaukomuntersuchung an der Spaltlampe empfiehlt, um jedes störende Nebenlicht peinlichst abzuhalten.

Seit Dezember 1916 kamen bei uns bis jetzt - Januar 1918 ungefähr 70 neue Fälle von klinisch sicheren Primärglaukomen an der Spaltlampe zur Untersuchung. Unter diesen waren 6 Fälle von akutem und inflammatorischem chronischen Glaukom vertreten, während der Rest einfache Fälle von Glaucoma simplex in den verschiedensten Stadien, einschließlich mit Glancoma absolutum, darstellte.

Von den letztgenannten Fällen von Glaucoma simplex zeigten ungefähr $80 \%$ diese oder jene der in Mitteilung 3 besprochenen Erscheinungsformen der glaukomatösen Pigmentverschiebung. Am häufigsten war die Sphincterpartie der Iris betroffen (Tafel II), am wenigsten der Ciliarteil, während die Pigmentverstäubung im Krausengebiete ungefähr die Mitte hielt. In bei weitem der Mehrzahl der Fälle war ferner die glaukomatöse Pigmentverschiebung über die ganze Tris völlig unregelmäßig verstreut, Sektoren- oder segmentförmige Anordnung, welche wir in Mitteilung 3 erwähnten, sahen wir in ca. 1 Dutzend Fällen, dagegen zeigten sämtliche Fälle von Glaucoma simplex und absolutum restlos eine Vermehrung der Pigmentpunkte auf der Hornhauthinterfläche verschiedensten Grades. Die Stärke der Pigmentbestäubung der Hornhauthinterfläche ging der klinischen Stärke der Symptome - wenigstens bei den hellbraunen Pigmentpunkten - nicht immer parallel, Während aber andererseits bei dunkelpigmentierter Iris die Pigmentpunkte in größerer Zahl und früher aufzutreten schienen als bei pigmentärmeren, waren speziell bei den absoluten Glaukomen beide Arten von Pigmentpunkten regelmäßig in großer Zahl zu finden.

Was die feinere Anordnung der Pigmentpunkte dabei betraf, so erschien hier regelmäßig das Bild, wie es in Mitteilung 3 beschrieben wurde. Es* zeigten sich entweder intakte hellbraune und dunkelbraune Pigmentzellen oder auch ihr freies Pigment info'ge von Pigmentzerfall.

Auch die auf der Irisoberfläche zu beobachtende Pigmentverstäubung sahen wir, soweit sie nicht durch höheres Alter im Sinne Höhmanns bedingt war, in ungefähr der Hälfte der Fälle.

Bei dunkelpigmentierter Tris war im allgemeinen der Nachweis der 
Pigmentverschiebung recht schwierig und nur in der Tiefe einiger nicht so stark pigmentierter Krypten und Lacunen vor allem mit Hilfe der Blauscheibe möglich. In $20 \%$ der Fälle von Glaucoma simplex gelang an der Spaltlampe der Nachweis der Pigmentverschiebung im Irisgebiete nicht.

Im Limbusbereiche und in der Nachbarschaft der Limbuscapillaren resp. der Limbuslymphgefäße sahen wir in 10 Fällen dunkelbraune Pigmentelemente in auffallend vermehrter Menge, ebenso auch entlang den perforierenden Ciliargefäßen. Weitere neue Besonderheiten zeigten sich nicht.

In $90 \%$ unserer Fälle war die Pigmentverstäubung doppelseitiz, vorausgesetzt, daß das Glaukom während der Beobachtungszeit klinisch einseitig war und es auch blieb.

Als typische und die Pigmentverschiebung recht deutlich doppelseitig zeigende Fälle seien aus dem Materiale, das uns zur Verfügung steht, folgende Fälle von doppelseitigem, klinisch sicherem Glaucoma simplex herausgegriffen.

Fall 1. Dorothea B., 64 Jahre (J.-N. 4278/16) hat typische Glaukomanamnese und die Beschwerden seit einem Jahr.

Der Befund ergibt:

$\mathrm{S}$ bds. $+1,0 \mathrm{D}=5 / 7$.

Bds. typische glaukomatische Exkavation, $\mathrm{R}$ mit atrophischer Ablassung, nasaler Verdrängung und Verengerung der Gefäße.

Druck bds. $28 \mathrm{~mm}$.

R nasale Einschränkung des Gesichtsfelds von oben her.

An der Spaltlampe bds. schwere typische Pigmentverstäubung unregelmäßig im Stroma, vor allem in Sphincter und Krause, aber auch auf der Irisoberfläche. Zahlreiche Hornhautpigmentpunkte. Beginnende stromale Atrophie.

Fall 2. Der 51 jährige Leopold H. (J.-N. 8086/17) sieht seit einem Jahre bds. Regenbogenfarben und hat bei einem Visus von $\mathrm{R}=5 / 35$ und $\mathrm{L}=5 / 7 \mathrm{E}$ bds. ty. pische glaukomatöse Exkavation mit Atrophie,

Druck $\mathrm{R}=33 \mathrm{~mm}$.

$$
\mathrm{L}=20 \mathrm{~mm} \text {. }
$$

Gesichtsfeld bds. nạsal eingeengt.

An der Spaltlampe zeigt sich bds. in Sphineter und Krause starke Pigmentverstäubung neben zahlreichen Pigmentpunkten der Hornhaut. Hier ebenfalls ausgesprochene stromale Irisatrophie.

F all 3. Der 85jährige Friedrich N. (J.-N. 769/17) hat früher immer gut gesehen, bemerkte aber seit einiger Zeit besonders links Abnahme des Sehens.

$\mathrm{S} R=5 / 10$ p. Gl. b. $n$.

$\mathrm{S} \mathrm{L}=$ Fingerz. v. d. A. (große Macula corneae).

Ophth. bds. sichere glaukomatöse Exkavation.

Druck $R=18 \mathrm{~mm}$.

' $\mathrm{L}=25 \mathrm{~mm}$.

Gesichtsfeld $\mathbf{R}$ von nasal unten eingeengt. Falle.

An der Spaltlampe bds. schwere Pigmentverschiebung wie im vorigen 
Doppelseitig zeigte sich die glaukomatöse Pigmentverschiebung an der Spaltampe bei einseitig manifestem Gla ucoma simplex vor allem bei diesen Patienten:

Fall 4. Die 70jährige A. B. merkte seit einem Jahre R Abnahme des Sehens, hatte aber sonst niemals Schmerzen oder Beschwerden.

$\mathrm{S} R=5 / 20$ p. Gl. b. n. (Cat. incip.)

S $L=5 / 15$ p. Gl. b. n. (Cat. incip.)

L A äußerlich und ophth. o. B.

R A außerlich o. B., aber ophth. tiefe randständige glankomatöse Exkavation mit deutlicher Atrophie.

Druck $\mathrm{R}=27 \mathrm{~mm}$.

$$
\mathrm{L}=24 \mathrm{~mm} \text {. }
$$

Das Gesichtsfeld zeigt links normale Ausdehnung, aber rechts starke nasale Einengung von oben und unten her.

An der SpaltI a m pe zeigt sich die Pigmentverschiebung an der stromatisch ziemlich dunkel pigmentierten Iris mit Hilfe der Blauscheibe bds. aussgesprochen vorhanden, $\mathbf{R}$ stärker als $\mathbf{L}$. Vor allem in den Krypten und Lacunen ist das Verhalten deutlich, .zum Teil daselbst auch auf der Oberfläche des Gewebes. Viele Hornhautpigmentpunkte bds.

Fall 5. Der 39 jährige Paul D. (5593/15) hatte niemals Glaukombeschwerden.

$\mathrm{S} R=-8,0 \mathrm{D}=5 / 15$.

$\mathrm{S} \mathrm{L}=5 / 7 \mathrm{E}$.

L ophth. $=\mathbf{n}^{\circ}$.

$R$ äuBerlich o. B., aber myopische Papille mit nasaler Verdrängung und rand ständiger Abknickung der Gefäße.

Gesichtsfeld $R$ nasal eingeengt.

Druck bds. $=22 \mathrm{~mm}$.

An der Spaltiam pe ist rechts viel stärker als links eine deutliche Pigmentverstäubung sowohl im Sphinkter als im Ciliarteile nachweisbar. Rechts fortgeschrittene Atrophie der Iris. Viele Pigmentpunkte in der Hornhaut beiderseits.

Fall 6. Bei der 58 jährigen Anna Sčh. (76/16) besteht $R$ ein typisches Glaucoma simplex bei einem Visus von Fgz. in $2 \mathrm{~m}$, Druck $45 \mathrm{~mm}$ und glaukomatöser Exkavation mit Atrophie.

L A Visus und ophth. normal.

$R$ nasale Einengung des Gesichtsfelds.

An der S paltla m pe $R$ stärker als $L$ ausgedehnte Pigmentverschiebung der Iris und auf der Irisoberfläche.

Speziell ein Fall von absolutem Glaukom ließ die Pigmentwanderung im stärksten Grade erkennen. Daneben bestand eine chronisch inflammatorische Reizung des Auges. Der Befund war dieser:

Fall 7. Bei der 54jährigen Emma W. (735/16) besteht $R$ seit einem Jahre Abnahme des Sehens und seit 4-6 Wochen völlige Erblindung. Sie hatte öfters Nebel- und Regenbogenfarbensehen.

Der Befund ergibt $L$ bei einem Visus von $-13,0=5 / 10$ und opth. sichtbarem Fundus myopieus für Glaukom keinen Anhalt.

$\mathrm{R}$ dagegen bei Amaurose typisches Bild des absoluten Glaukoms. Die Sachs. sche Lampe ergibt von allen Seiten guten Lichtschein.

An der Spaltiampe $L$ normaler Befund.

$\mathbf{R}$ schwerste Pigmentverstäubung in und auf der Iris, im Kammerwasser und auf der Hornhauthinterfläche. Die Iris ist stark atrophisch, ihre Bälkchen haben ein strohiges Aussehen und die Gefäße sind zum Teil verödet. 
Bei unseren Fällen von chronischem entzündlichem Gla u kom zeigten sich stets mehr oder weniger Pigmentstaubspuren im Kammerwasser und stärkere Pigmentverschiebung in der Iris neben zahlreichen Pigmentpunkten der Cornea, ebenso bei unseren sämtlichen Fällen von akutem Glaukom. Die Pigmentverschiebung saß überall unregelmäßig in der Iris und bot außer den in Mitteilung $\mathbf{3}$ beschriebenen Erscheinungen bei diesen Fällen nichts Neues. Bei sämtlichen Fällen war auch auf dem anderen Auge, aber weniger ausgeprägt, Pigmentverschiebung vorhanden.

Die Krankengeschichte des einen Falles von akutem Glaukom sei hier noch angeführt.

Fall 8. Die 57jährige Pauline W. (J.-N. 913/17) litt seit 2 Jahren viel an Kopfschmerzen und Regenbogenfarbensehen vor dem rechten Auge. Seit 8 Tagen $\mathrm{R}$ schwerste Augenentzündung.

$\mathrm{L} \mathrm{S}=+2,0 \mathrm{D}=5 / 5 ; \mathrm{O}=\mathrm{n}$.

R $\mathbf{S}=$ Hdbw. Gl. b. n.

Typisches Bild des akuten Glankoms.

Druck $\mathrm{R}=70 \mathrm{~mm}$.

$\mathrm{L}=15 \mathrm{~mm}$. Gesichtsfeld normal.

An der Spaltlam pe $L$ normaler Befund.

$\mathrm{R}$ massenhafte Pigmentzellen und Pigmentstaub im Kammerwasser und auf der Hornhauthinterflàche. Auf die Iris zunächst kein deutlicher Einblick.

Acht Tage nach der Operation sieht man dagegen in der atrophischen rechten Iris ausgedehnte Pigmentverschiebung im Sphinctergebiete und ophthalmoskopisch eine fortgeschrittene glaukomatöse Exkavation.

Des weiteren beobachteten wir nun eine größere Anzahl von Augen。 die zwar klinisch keine Glaukomzeichen darboten, deren Anamnese aber in einem Teile der Fälle verdächtig war, während an der Spaltlampe diese oder jene Erscheinungsform der Pigmentverschiebung einoder doppelseitig mehr oder weniger ausgesprochen bestand.

Aus diesen 9 Fällen von Präglaukom seien, um Wiederholungen zu vermeiden, nur diese 3 recht typischen herausgegriffen:

Fall 9. Die 46jährige Marta G. hatte niemals Glaukombeschwerden, und ließ bei der Untersuchung klinisch bds. $\mathrm{S} E=5 / 7$ und normalen ophth. Befund erkennen.

An der Spaltlampe zeigte sich aber bds. typische Pigmentverschiebung geringen Grades vor allem im Sphineter und Krause, vereinzelt aber auch im Ciliarteile und auf der Irisoberflache. Viele Hornhautpigmentpunkte.

Der Bjerru m war in beiden Augen der wegen Presbyopie uns konsultierenden Patientin negativ. Druck bds. $24 \mathrm{~mm}$.

Fall 10. Ähnlich verhielt es sich bei dem 62jährigen Karl E. (5207/16).

Der Befund ergibt bds. Cat. incip., ist sonst aber sowohl ophth. wie dem Gesichtsfeld nach normal. Speziell schien Bjerrum negativ und der Druck ben trug bds. $26 \mathrm{~mm}$.

Fall 11. Die 58 jährige Amalie F. (5470/16) klagte seit einem Jahre bds. über Anfälle von Nebelsehen und Kopfschmerzen.

R S $=5 / 20$, Gl. b. n. (Cat. incip.)

L S $=+3,0 \mathrm{D}=5 / 5$. 
Ophth. und Gesichtsfeld bds. sonst normal.

An der Spaltlampe bds. Vermehrung der Hornhautpigmentpunkte und beginnende Pigmentverschiebung hier und da sektorenförmig im Sphinetergebiet. Daselbst beginnende leichte stromale Atrophie.

Fall 12. Marie K., 61 Jahr (3619/16), hatte niemals Beschwerden.

Bds. $+2,0 \mathrm{D} \mathrm{S}=5 / 10$.

Ophth. bds. muldenförmige, fast randständige Exkavation ohne Abknickung aber mit leichter nasaler Verdrängung der Gefäße.

Druck R $23 \mathrm{~mm}$, L $20 \mathrm{~mm}$.

Gesichtsfeld bds. normal, Bjerrum negativ.

An der S paltlampe $R$ stärker als $L$ vermehrte Pigmentpunkte der Cornea und Andeutung von Pigmentverstäubung segmentartig in und auf dem Krausengebiet. Keine stromale Atrophie.

Wir verfügten auch im vergangenen Jahre wieder über einige Fälle von Katarakt, welche die Pigmentverschiebung an der Spaltlampe darboten, sonst aber keine Zeichen von Glaukom klinisch erkennen ließen.

Fall 13. Der 57jährige Wilhelm B. (J.-N. 890/16) kommt links mit einer reifen Katarakt in die Klinik, zeigte auf diesem Auge richtigen Lichtschein und Projektion bei einem Drucke von $18 \mathrm{~mm}, \mathrm{R}$ dagegen beginnende Katarakt bei einem Visus von $5 / 35$ und einem Drucke von $20 \mathrm{~mm}$. Ophth. R normaler Befund, aber an der Spaltlampe bds. fortgeschrittene Pigmentverschiebung. R Gesichtsfeld wegen der beginnenden Katarakt nicht eindeutig. Nach der Extraktion linker Fintergrund $\mathrm{O}=\mathrm{n}$.

Fall 14. Völlig analog verhielt sich die Sache bei der 66jährigen Anna M. (J.-N. 756/16), doch war hier die Pigmentverschiebung nur auf das rechte Auge, das die reife Katarakt besa $\beta$, beschränkt. Auch nach der Extraktion war der Hintergrund normal.

Fall 15. Der 77jährige Karl K. $(5827 / 16)$ hat $\mathrm{R}$ reife Katarakt mit richtigem Lichtschein und $\mathrm{L}+3,5 \mathrm{D} \mathrm{S}=5 / 10$.

Ophth. L vielleicht randständige physiologische Exkavation ohne Abknickung der Gefäße. Druck bds. $20 \mathrm{~mm}$.

Gesichtsfeld L normal, Bjerrum negativ.

An der Spaltlampe $\mathbf{R}$ stärker als $L$ beginnende Pigmentverschiebung im Sphincter und Vermehrung der Hornhantpigmentpunkte. Leichte stromale Irisatrophie.

Damit kommen wir nun zu derjenigen eigentümlichen Gruppe der ,Bindeglied-“ oder ,Brückenfälle", die den Übergang zur erst genannten Gruppe in die zweite darstellen dürften.

$\mathrm{Zu}$ diesen eigentümlichen Fällen, von denen wir bereits in Mitteilung 3 vier Fälle als Beleg für den vermuteten Zusammenhang zwischen beiden Erscheinungsformen des Glaukoms anführen konnten, haben wir in dem vergangenen Jahre 6 neue beobachtet und wir möchten daher nicht verfehlen im Interesse der außerordentlichen Bedeutung, die wir dieser Gruppe für die gesamte Auffassung und die theoretische Deutung bezüglich der Glaukomgenese beimessen, auf diese Fälle ausführlich zu sprechen zu kommen.

Zunächst beobachteten wir 2 Fälle, die wieder für sich gewisser- 
maßen den Übergang eines Präglaukoms in einen Brückenfall darstellen, und zwar insofern, als trotz zweifelhafter An amnese nur allein das Bjerrumsche Zeichen neben der Pigmentverschiebung bestand, während sonst jedoch keinerlei Glaukomzeichen objektiv nachweisbar waren.

Der erste der beiden Fälle war dieser:

Fall 16. Der 30 jährige André M. (7493/17) klagt seit 2 Jahren bds. über Anfälle von Regenbogenfarbensehen.

$\mathrm{R} \mathrm{S}=5 / 15$. Gl. b. n.

$\mathrm{L} \mathrm{S}=-2,0 \mathrm{D}=5 / 35^{\circ}$. Gl. b. n.

Ophth. bds. ziemlich große physiologische Exkavation, die aber nicht randstandig ist. Papillenfarbe and Hintergrund normal.

Druck bds. $18 \mathrm{~mm}$.

Gesichtsfeldaußengrenzen bds. absolut normal, dagegen bds. Andeutung von Ejerrum nach oben und unten.

An der Spaltla m pe zeigt sich bds. an der stromal ziemlich dicht pigmentierten Iris in den Krypten und Lacunen mittels der Blauscheibe ausgesprochene Pigmentverschiebung, vor allem im Sphinctergebiete, dagegen noch keine stromale. Atrophie. Unfalls.

Fall 17. Der 56 jährige L. W. $(4623 / 16)$ hat R Anophthalmus infolge früheren

L niemals Beschwerden gehabt.

$\mathrm{L} \mathrm{ES}=5 / 20$ (Cat. inc.).

Ophth. L absolut normal, Druck $23 \mathrm{~mm}$, Gesichtsfeldaußengrenzen normal. Bjerrum ausgesprochen vorhanden.

An der Spaltlampe hier und da beginnende Pigmentverschiebung. im Sphinetergebiet und zum Teil auch in der Krause.

Epikritisch können wir hier hinzufügen, daß zwar das positive, resp. angedeutete Bjerrumsche Zeichen neben der allerdings unsicheren Anamnese - vor allem im ersten Falle - sehr zugunsten der Annahme eines bestehenden und im Frühstadium befindlichen Glaukoms sprach. Angesichts des Fehlens jeder weiteren objektiven Glaukomzeichen bedeutete gerade hier die Verbindung der Pigmentverschiebung mit dem positiven Bjerrumschen Zeichen einen wichtigen diagnostischen Fingerzeig in der Richtung, daß die Vergrößerung des blinden Fleckes im Sinne Bjerrums als ein. bei diesen Fällen in der Entwicklung begriffenes Glaukom gedeutet werden mußte, welches gerade anfing, klinisch manifest zu werden und sich zunächst erst nur der verfeinerten Bjerrumschen Methode erschloß.

Bei den folgenden beiden Fällen von Katarakt war klinisch eine auch nicht annähernd gesichert erscheinende Glaukomdiagnose zu stellen; weder aus dem Drucke, noch aus der Projektion oder dem klinischen äußeren Augenbefunde - abgesehen von der Spaltlampe konnte ein objektives Glaukomzeichen ermittelt werden. Aber nach der Extraktion der Katarakt bestätigte die Augenspiegeluntersuchung dievorher an der Nernstspaltlampe aus der 
bestehenden Pigmentrerschiebung gestellte Glaukomdiagnose.

Fáll 18. Der 80 jährige August A. (J.-N. 508/16) war L auswärts starextrahiert und hatte mit Korrektur bei normalem ophth. Befunde einen Visus von $5 / 35$.

$R$ reife Katarakt mit richtigem Lichtschein und Projektion.

Druck bds. $18 \mathrm{~mm}$.

L Gesichtsfeld normal.

An der Spaltlampe L sekundüre Pigmentverschiebung nach postoperativer Iritis.

$\mathrm{R}$ schwere Pigmentverschiebung im Sphincter- und Krausengebiet mit deutlicher stromaler Atrophie.

Nach Abschluß der postoperativen Nachbehandlung und Diszission des Nachstars L zeigt sich bds. eine typische glaukomatöse Exkavation mit Atrophie und engen Gefaßen.

Fall 19. Bei dem 75jährigen Wilhelm R. (J.-N. 497/17) war seit über einem Jahre das Sehen auf beiden Augen immer schlechter geworden.

Der Bêfund ergibt:

Bds. Cataracta matura mit gutem Lichtschein und richtiger Projektion. Druck bds. $18 \mathrm{~mm}$. Bds. äußerlich o. B.

An der Spaltlampe bds. fortgêschrittene Pigmentverschiebung der Iris in Sphincter und Krause neben fortgeschrittener richtig ,strohiger" Atrophie der Stromas, ferner starke Vermehrung der Hornhautpigmentpunkte.

Nach Extraktion und Abschluß der Nachbehandlung ergibt der Augenspiegel bds. eine typische glaukomatöse Exkavation mit beginnender Atrophie und engen Gefäßen.

Bei diesen letzten beiden Fällen war so recht bemerkenswert, daß mit unseren bisherigen Hilfsmitteln keine Spur eines bestehenden Glaukoms nachgewiesen werden konnte und erst die Spaltlampe das Bestehen oder mindestens die Entwicklung eines solchen annehmen ließ, bis nach Extraktion der Linse tatsächlich durch den Nachweis einer glaukomatösen Exkavation der Beweis dafür erbracht werden konnte, daß die an der Spaltlampe beobachteten Pigmentveränderungen für Glaukom spezifisch waren.

Besonders eigentümlich lag die Sache auch bei

Fall 20. Ein 65 jähriger Patient kommt mit Cataracta senilis intumescens auf dem linken Auge und mit normalem rechten Auge zur Untersuchung. Es findet sich $\mathrm{X}$ ein Druck von $35 \mathrm{~mm}$ bei richtiger. Projektion, aber an der Spaltlampe stärkster Pigmentverschiebung. Das Auge war völlig reizlos und der Patient hatte schon seit Jahren, noch vor Einsetzen der durch die Katarakt bedingten Sehverschlechterung über Regenbogenfarbensehen geklagt. Das rechte Auge erschien auch an der Spaltlampe normal, während L die besagte schwere Pigmentverschiebung bei relativ enger Vorderkammer erkenntlich war. Da Patient bis jetzt die Operation verweigerte, ferner auch das rehte Auge reizlos blieb, konnte die Glaukomdiagnose vorläufig auch noch nicht bestätigt werden. Auf dem rechten Auge bestand ein Druck von $18 \mathrm{~mm}$ und negativer B jerr u m, auch bei einer Nachuntersuchung ein Vierteljahr später wurde derselbe Befund erhoben.

Ganz ähnlich lagen noch 2 weitere Fälle unserer Praxis.

Wenn somit bei diesen Fällen ebenfalls noch kein klinischer Nachweis des Glaukoms - abgesehẹn vọn der Drucksteigerung - möglich 
war, so war doch hier wenigstens neben der Pigmentverschiebung das Glaukom klinisch höchst wahrscheinlich gemacht.

Gegenüber allen diesen an sich schon sehr denkwürdigen Fällen demonstriertinden folgenden beidenFällen tatsächlich das a n derS paltla m pe diagnostizierte präglaukomatöseStadium den Ưbergang in das klinisch manifeste und nachweisbare Primärglaukom:

Fall 21. Die 72 jährige Henriette Sch. (J.-N. 721/16) klagt seit einem Jahre über Sehverschlechterung auf einem Auge; seit 4 Wochen Schmerzen auf diesem Auge. War fruher niemals augenkrank und hatte auch $\mathrm{L}$ niemals Beschwerden.

Der Befund am 4. XII. 16 ergibt:

R $\mathrm{S}=$ Lichtschein $\mathrm{f}$. kl. Fl. Projektion von oben und innen falsch.

$\mathrm{LS}=-1,5 \mathrm{D}=5 / 10$.

$\mathrm{R}$ typisches Bild des absoluten Glaukoms bei $20 \mathrm{~mm}$ Druck und schwerster Pigmentverstäubung im Irisstroma, im Kammerwasser und auf der Hornhauthinterfläche. Iris sehr stark atrophisch. L A äußerlich und ophth. völlig o. B. Gesichtsfeld normal, Bjerrum negativ.

An der Spaltlam pe besteht auch L im Sphincter-, Krausen- und Ciliargebiete der Iris fortgeschrittene Pigmentverschiebung, ebenso auf der Irisoberfläche und auf der Hornhauthinterfläche. Das Irisstroma selbst ist an den Stellen der Pigmentverschiebung ganz leicht im Beginne der Atrophie.

Am Tage nach der Aufnahme wurde das rechte Auge enucleiert und Patientin einige Tage danach entlassen.

Im Marz ersshien Patientin wieder mit der Angabe, daB sie auf dem linken Auge seit 14 Tagen háufig Anfálle von Regenbogenfarbensehen bemerkt habe.

Der Befund ergibt jetzt denselben Visus, keine Exkavation, aber deutlich einen positiven Bjerrum bei einem Drucke von $30 \mathrm{~mm}$. Die Außengrenzen des Gesichtsfeldes zeigten sich noch normal.

An der Spaltiampe noch derselbe Befund der Pigmentverschiebung.

Im September 1917 kam Patientin wieder mit der Angabe von Regenbogenfarbensehen und Kopfschmerzen bei einem Drucke von $32 \mathrm{~mm}$, weiter vergrößertem Bjerrum und beginnender nasaler Gesichtsfeldeinengung von unten innen her. Der Visus war noch derselbe geblieben, aber ophthalmoskopisch waren die Gefäße jetzt sehr deutlich nasal verdrängt und die Exkavation war temporal randständig geworden unter beginnender Abknickung eines der Papillengefäße.

Der zweite Fall dieser Art betraf die 42jährige Emma R. (427/17).

Fall 22. Es handelte sich hier um dieselbe Patientin, die seinerzeit (Mitteilung 3) wegen Presbyopie in die Sprechstunde gekommen war, woselbst klinisch normaler Befund, aber beiderseits ein fortgeschrittenes Präglaukom an der Spaltlampe diagnostiert wurde.

Damals hatte sie einige Tage nach der Untersuchung einen typischen Glaukomanfall auf dem linken Auge; welches dann operiert wurde und zur Abheilung kam.

Dreiviertel Jahr danach $\mathrm{kam}$ sie wieder zu uns mit einem typischen schweren Glaukomanfalle auf dem rechten Auge, einem Drucke von $\mathrm{R} 28 \mathrm{~mm}$ und stärkster Pigmentverstäubung auf der Hornhauthinterfläche und im Kammerwasser. Nach Abheilung des Anfalls bestand ophth. noch keinerlei glaukomatöser Befund.

Diese zuletzt geschilderten beiden Fälle des direkten Übergangs eines Präglaukoms in ein klinisch manifestes Glaukom dürften weiterhin geeignet sein, unsere Anschauung betreffs der primären und auch ätiologischen Bedeutung der geschilderten. Pigmentveränderungen für 
die Entstehung und vor allem auch für die Frühdiagnostik des Primärglaukoms recht wesentlich zu stützen. Diese Fälle stellen wieder zwei typische Bindeglied- oder Brückenfälle dar, wie wir sie schon früher für unsere Schlüsse ins Treffen führen konnten.

Abgesehen von der Wichtigkeit der Pigmentversehiebung für die Frühdiagnose des Primärglaukoms an der Spaltlampe, die wieder aus den geschilderten letzten beiden und vor etzten drei Fällen zur Genüge hervorging, wollen wir nicht verfehlen, hier noch einmal auf die Wichtigkeit der an der Spaltlampe erkenntlichen frühzeitigen stromalen Atrophie der von der Pigmentverschiebung betroffenen Iristeile hinzuweisen. Trotz noch gut erhaltener Contractilität der einzelnen feinsten Muskelbälkchen leidet das normale, teils wollige, teils schwammige Aussehen dieser Gebilde im Bereiche der Pigmentverschiebung mitunter schon recht deutlich, so daß auch gerade das Zusammentreffen dieser frühzeitigen stromalen Atrophie mit der beginnenden oder fortgeschrittenen Pigmentverschiebung für die Frühdiagnóstik des Primärglaukoms von besonderer Bedeutung erscheint.

Auch 2 Fälle von Hydrophthalmus bei einem 8- bzw. 10jährigen Kinde konnten wir an der Spaltlampe beobachten und bei ihnen das Bestehen einer fortgeschrittenen Pigmentverschiebung feststellen, was an den ausgeschnittenen Irisstücken dann in der in Mitteilung 3 mitgeteilten Weise wieder mikroskopisch bestätigt werden konnte.

Auch bei den übrigen oben erwähnten Fällen, soweit operatives Untersuchungsmaterial zu erlangen war, fanden wir dieselben Veränderungen, welche wir früher als das mikroskopisch-anatomische Bild der beim Primärglaukom in der Iris festzustellenden Pigmentveränderungen mitgeteilt haben.

Bei den Untersuchungen des letzten Jahres legten wir besonderen Wert darauf, durch Anwendung der Entfärbungsmethode von Weigert-Pal das hellbraune Stromapigment zu entfärben, so daß bei Lithionearminfärbung und Ölimmersiom nur das dunkelbraune Pigment und seine Zerfallsteile in Naturfarbe zu sehen waren.

In vielen Fällen zeigte sich da wiederum das Pigmentepithel mehr oder weniger im Zerfalle, während freie Pigmentkörnchen von polyedrischer oder mehr rundlicher Form, teils intakt, teils zerfallen, vor allem in der Gegend der hinteren Grenzschicht und dieselbe durchbrechend allenthalben auch in den vorderen Stromalagen zu finden waren, woselbst sie entweder die Wandung der perivasculären Lymphgefäße der Iris bevorzugten oder auch mehr vereinzelt frei in den intrastromalen Gewebslücken ${ }^{1}$ ) selbst auftauchten. Auch in der Gegend der

1) Inzwischen sind diese Befunde durch den Fall Thomsens anatomisch bestätigt worden (H. Thomsen, Anatom. Unters. eines akut. inflammat. Glauk. Klin. Mon. f. A. 60, März 1918). 
vorderen Grenzschicht, auf des Irisoberfläche sowie in der Nachbarschaft der "Irisoberflächenporen" war das ebenfalls hier und da der Fall.

Durch wechselnde Einstellung des Mikroskoptubus kann man sehr gut Kunstprodukte, wie durch die Schneide zerstreute Pigmentpartikel usw. von dem eigentlichen ,Glaukompigment" unterscheiden. Während die ersteren, wie die entsprechende Einstellung beweist, stets auf der Oberfläche der Schnitte ganz unregelmäßig gelegen sind, ist das freje Glaukompigment bei wechselnder Einstellung stets in allen Lagen zu finden, so daß dann hier von Kunstprodukten nicht gesprochen werden kann.

In einigen Fällen war der Nachweis der freien Pigmentkörnchen recht schwierig und dieselben nur in sehr geringer Zahl frei im Irisstroma nachweisbar. Erst bei hellster Beleuchtung und folgender starker Abblendung mit Ölimmersion und Okular 4 konnte man die Partikelchen erkennen. Das Bild war jedoch von den übrigen Fällen mit positivem Befund nur graduell verschieden und etwas geringer ausgeprägt, während kein einziger Fall freie Pigmentspuren vermissen ließ.

Anhangsweise sei nun noch zweier Fälle gedacht, bei denen die glaukomatöse Pigmentverstreaung im Irisstroma bei Diabetes auftrat und bei dem einen Falle infolge Fehlens kliniseher Glaukomsymptome ein Präglaukom anzeigte, bei dem anderen jedoch ein klinisch manifestes Glaucoma simplex begleitete.

Mit besonderer Sorgfalt haben wir bisher an der Nernstspaltlampe Dia betiker, bezugsweise das Verhalten ihres Irispigments untersucht. Bekanntlich hat das Pigmentepithel bei Diabetes eine in seiner Konsistenz mehr oder minder herabgesetzte Besehaffenheit und sitzt ziemlich locker. Der Zellverband ist gelockert, das Pigment dabei im allgemeinen etwas weniger fest an die Zelle gebunden. Uhthoff $u$. a. haben auf diese Tatsache hingewiesen. Man beobachtet dabei das geschilderte Verhalten der Pigmentmaceration in vivo bei Diabetes vor allem nach geringfügigen operativen Eingriffen und kann das Phänomen der dabei auftretenden verschieden ausgeprägten Pigmentverstreuung auf der Iris und ihrer Nachbarschaft gelegentlich zu sehen bekommen.

Trotz stärkster Vergrößerung konnten wir nun bei einem größeren Materiale von Diabetikern - für dessen Überlassung ich den Herren Dr. Kaufmann und Dr. Pollag an der hiesigen medizinischen Universitätsklinik zu großem Danke verpflichtet bin - ohne daß operative Eingriffe an dem Auge der betreffenden Patienten vorausgegangen waren, in vivo niemals deutliche Zerfallsprozesse im Pigmentepithel feststellen, speziell keinen freien intrastromalen Pigmentstaub. Mitunter fand sich nur eine relativ frühzeitige Pigmentverstreuung in der Nähe des Pupillarrands, wie sie von Höhmann beschrieben wurde. 
Offenbar ist mithin der diabetische Pigmentzellzerfall da, wo er erfolgt, eine Abstoßung vorwiegend intakter Zellen und kein Pigmentzerfall in Staub in unserem Sinne. Ferner dürfte die diabetische Pigmentzelldissolution und -maceration an das Vorausgehen mehr oder weniger eingreifender operativer Maßnahmen geknüpft sein; wie ja auch die Literatur hierüber zeigt.

Wenn auch beim Diabetes neben der leichteren Ablösungsmöglichkeit der Pigmentzellen das eigentliche Pigment selbst vielleicht etwas lockerer an die Zellen gebunden ist, so dürften doch beim glaukomatösen Pigmentzellzerfalle andere biochemische Veränderungen der Zellen bestehen, um den für Glaukom typischen Pigmentzerfall zu veranlassen, Veränderungen, deren Natur ihrem Wesen nach vorderhand dunkel bleibt, wenn auch Einflüsse von seiten des sympathischen Nervensystems dabei wahrscheinlich sind.

Was nun die beiden erwähnten Fälle von glaukomatöser Pigmentverschiebung bei Diabetes selbst betrifft, so zeigte die Spaltlampe bei dem einen der beiden Fälle ein typisches Präglaukom, bei dem anderen ein klinisches Glaukom mit fortgeschrittener Pigmentverschiebung.

Fall 23. Der 30jährige G. K. (8241/17) leidet seit 2 Jahren an Diabetes und hat $2-3 \%$ Zucker.

Bds. $=-1,0 \mathrm{D} \mathrm{S}=5 / 5$.

Ophth. zeigt sich $\mathbf{R}$ absolut normaler Befund, dagegen $\mathrm{L}$ in der Gegend der Macula einige streifige weiße Degenerationsherde ohne Blutungen.

An der Spaltlam pe findet sich bds. fortgeschrittene ziemlich gleichmäßig die ganze Iris betreffende ausgesprochene Pigmentversohiebung mit zahlreichen Pigmentpunkten der Hornhauthinterfläche.

Fall 24. Bei einem 17jährigen Mädchen, das monatelang Zucker hatte, infolge der Behandlung aber jetzt zuckerfrei war, findet sich bds. eine beginnende diabetische Katarkt, ein Visus von $5 / 50$ und eine ausgesprochene glaukomatóse Exkavation mit beginnender Atrophie der Papillen, leicht nasaler Gesichtsfeldeinschrankung und bds. $28 \mathrm{~mm}$ Druck. Falle.

An der Spaltlampe bds. Pigmentverschiebung ungefähr wie im vorigen

Bei beiden Fällen fand sich, was bemerkenswert erscheint, kein Dissolutionsprozeß intakter Zellen in höherem Maße, dagegen ausgesprochen staubförmiger Pigmentzerfall. Auch auf der Irisoberfläche, vor allem in der Nähe des Pupillarsaums, ist beiderseits keine stärkere Verstreuung von dunkelbraunen Pigmentelementen sichtbar. In beiden Fällen bestand wohl kaum ein Zusammenhang zwischen der glaukomatösen Pigmentverschiebung und der leichteren Dissolutionsmöglichkeit hauptsächlich intakter Pigmentzellen infolge des bestehenden Diabetes.

Anhangsweise möchte ich nun noch einmal auf folgenden, schon in Mitteilung 3 berührten Punkt zu sprechen kommen.

Es dürfte nämlich bei denjenigen Fällen von Iritis, bei denen es im Verlaufe der Entzündung leichter als bei anderen Augen mit gleichen 
Veränderungen zu sekundärer Drucksteigerung und glaukomatösen Erscheinungen zu kommen vermag, eine schon vor Ausbruch der Iritis längst bestehende Pigmentverschiebung, die ihrerseits eine ,glaukomatöse Disposition" bedingte, mit im Spiele gewesen sein. Wir beobachteten einige Iritisfälle dieser Art mit Pigmentverschiebung in mittleren und älteren Jahren, bei denen die Pigmentversehiebung doppelseitig, aber die Iritis einseitig war. Sollte hier nicht die Iritis für die durch die Pigmentverschiebung geschaffene Glaukomdisposition dasjenige Plus dargestellt haben, was nötig war, um das schon latente Glaukom klinisch manifest zu machen? Oder kann nicht auch bei Fehlen einer Iritis in dem einen der oben genannten Fälle die Cataracta senilis intumescens dieses Plus an dem durch die Pigmentverschiebung glaukomdisponierten Auge ausgelöst haben? Wie dem auch sei, auf jeden Fall scheint mir die Frage dringend wert, allseitig genauer mit dor Nernstspaltla mpe nachgeprüft zu werden. Vielleicht ergiebt sich da für manches mit Iritis levis irgend welcher Natur und frühzeitigen Sekundärglaukomerscheinungen behaftetes Auge in einer bis dahin unentdeckten Pigmentversehiebung des präglaukomatösen Stadiums der Schlüssel zum Verständnisse der relativ frühzeitigen Drucksteigerung bei $\mathrm{Ab}$ wesenheit von Seclusio und ähnlichen Komplikationen, die ihrerseits das Glaukom hätten bedingen können.

So recht evident erschien mir die glaukomatöse Disposition infolge Pigmentverschiebung bei einem 23jährigen Dienstmädchen M. G. (Fall 25).

Diese Patientin hatte auf dem linken Auge seit längerer Zeit eine mit klümpchenförmigen Beschlägen einhergehende tuberkulöse Tritis und opth. auf diesem Auge eine glaukomatöse Exkavation mit Atrophie, obwohl die Irirtis noch gar nicht lange bestanden hatte. Der Druck betrug $40 \mathrm{~mm}$, gegen $20 \mathrm{~mm} \mathrm{R}$.

R klinisch völlig normalér Befund.

An der Spaltlam pe bds, fortgeschrittene Pigmentverschiebung, die sich $L$ den iritischen Veränderungen hinzugesellte.

$\mathrm{Ob}$ in diesem Falle die frühzeitige Drucksteigerung wirklich nur auf Konto der Iritis zu setzen war, erschien recht zweifelhaft, abgesehen von der Jugend der Patientin. Weitere Fälle dieser Art müssen abgewartet werden, um auch hier größere Klarheit in diese teilweise noch recht dunklen ätiologischen Verhältnisse zu werfen. ${ }^{1}$ )

Betonen möchte ich an dieser Stelle noch einmal ganz besonders,

1) Vielleicht kann auch eine jahrelang bestehende Verwachsung der Iris mit der Hornhaut, ein Leukoma adhaerens, durch die jahrelange Dehnung der Iris resp. des Pigmentepithels zu trophischen Störungen im Pigmentepithel und mit dem Wege hierüber eventuell auch zu Pigmentverschiebung führen, welche ihrerseits wiederum die Glaukomdisposition bei diesen Augen steigert, wenn nicht gar auch - wenigstens bei einem Teile der Fälle — bedingt. Wir sahen einige solcher Augen mit stark überdehnter Iris bei Leukoma adhaerens, bei der es zum Unterschiede gegen die auch an der Spaltlampe vollig gesunde andere Iris zu Pigmentverschiebung und klinisch sicherem Sekundarglaukom gekommen war, obwohl eine Seclusio usw. fehlte. 
daß die an der Nernstspaltlampe nach allen unseren Beobachtungen so gut wie ziemlich sichere Möglichkeit einer Frühdiagnose des Primärgla ukoms verdient, in. weitesten. Kreisen der Fachgenossen nachgeprüft zu werden. Wir haben in den glaukomatösen Pigmentveränderungen trotz Fehlens jeder weiteren Glaukomsymptome ein Mittel an der Hand, zum mindesten die glaukomverdächtigen Fälle schon vor dem Auftreten klinischer Erscheinungen festzustellen. Kommt dazu noch eine verdächtige oder zweifelhafte Anamnese wie gelegentliches Regenbogenfarbensehen, Bjerrum oder ähnliches ohne nur irgendwie ersichtlichen klinischen Grund, so erscheint uns die von uns aufgestellte Forderung in jeder Beziehung doppelt gerechtfertigt.

Wir erwähnten schon einmal ganz kurz, daß s y m pathische Einflüsse für die Entstehung der glaukomatösen Pigmentverstäubung von kausaler Bedeutung sein dürften und speziell für das Glaucoma simplex vielleicht in trophoneurotischen Störungen des Pigmentepithels zu suchen sind. Auf den letzteren Punkt hinzuweisen, hatten wir schon in Mitteilung 3 Gelegenheit gehabt. Dïese Fragen stehen nun wiederum zu einer weiteren Veränderung der Iris, der Heterochromie, in engster Beziehung.

So berichtete schon Galecowsky über 7 Fälle von Heterochromie der Iris und glaubte für alle diese Fälle einen Zusammenhang mit Sympathicuserkrankungen annehmen zu müssen, während Dehtleffsen bei 9 Fällen von Heterochromie eine gleichseitige Sympathicusparese feststellen konnte. In ähnlichem Sinne wie Galecows k y ä ßerte sich Bistis. Auch dieser Autor glaubt als ursächliches Moment der Heterochromie irgendeine Affektion des Sympathicus beschuldigen $\mathrm{zu}$ müssen und kommt in dieser Auffassung der Dinge sogar dahin, den in der Heterochromie zum Ausdruck kommenden Pigmentschwund der Iris gewissermaBen als viertes Symptom des bekannten Hornersehen Komplexes aufzufassen. Auch experimentell konnte er durch Exstirpation des obersten Halsganglions des Sympathicus bei Versuchstieren deutliche Depigmentation der Iris ${ }^{1}$ ) hervorrufen. Eine ähnliche Erklärung der Heterochromie als trophoneurotische Störung im Sympathicusgebiete des betreffenden Auges machte schon $L$ u tz namhaft, ferner Majou,Scalinci, Seeligmüller, Mendel, v. Herrenschwand ${ }^{2}$ ) u.a.

1) Interessant ist neuerdings die Beobachtung von Chotzen und Kutznitzky, welche durch Bestrahlung der Tris mit ultraviolettem Tichte ebenfalls Depigmentationen daselbst beobachten konnten. Bezüglich der Lichtbedeutung bei der Pigmentverschiebung finden wir hier gewissermaßen die Bestätigung unserer in Mittlg. 3 ausgesprochenen Mutmaßung, daß auch die jahrelange Bestrahlung der Iris mit nicht in der Linse gefiltertem Sonnenlichte vielleicht ätiologisch für das Bestehen der Pigmentverschiebung mit bedeutsam sein dürfte.

2) Nach diesem Autor nur dann, wenn die Störung zu oder vor der Zeit der Pigmententwicklung einsetzt. 
Wenn auch Metzner und Wölfflin nicht bestreiten, daß infolge einer Affektion des Sympathicus einmal deutlicher Pigmentschwund in der Tris der betreffenden Seite eintreten kann, obwohl sie auch experimentell das Eintreten der Depigmentation bei ihren Versuchstieren stets vermissen mußten, so konnte auch von Saenger, Hertel, Mohr, Reinhardt und Franke die Erscheinung der Irisdepigmentation nach Sympathicusalterationen niemals beobachtet werden. Auch Wessely, ferner Elschnig, Liebrecht, de Lapersonne, Jickeli, Roche, Russell u. a. erwähnten bei ihren Fällen von traumatischer Läsion des Sympathicus keinerlei Pigmentveränderungen dẹr Iris.

Wenn somit aus den bisher vorliegenden Ergebnissen der gesamten Literatur noch nicht einwandfrei feststeht, ob nun tatsächlich die bei der Heterochromie zu beobachtende Depigmentation der Iris allein als ein trophoneurotisches Symptom aufgefaßt werden muß, so hatten wir schon in Mitteilung 3 die Vermutung ausgesprochen, daß die glaukomatöse Pigmentverschiebung vielleicht auch als ein analoger Ausdruck einer sympathischen Störung am Pigmentepithel der Iris ${ }^{1}$ ) angesprochen werden dürfte. Als weitere Stütze für diese Auffassung wollen wir noch einige Krankengeschichten mitteilen, die einen solchen $\mathrm{Zn1}$ sammenhang zwischen Sympathicusveränderung und Pigmentverschiebung einerseits und zwischen Heterochromie und Glaukom andererseits wahrscheinlich machen, so daß man den Kreis Sympathicusveränderung -Heterochromie-Glaukom resp. glaukomatöse Pigmentverschiebung als in weiterem Sinne in sich geschlossen bezeichnen darf.

Als Beleg für die vermutete Beziehung zwischen Sympathicusläsion und Pigmentverschiebung seien hier 3 Fälle mitgeteilt, die für die Auffassung der Dinge nach dieser Richtung hin bemerkenswert erscheinen.

F all 26. Der 35 jährige Emil F. (J.-N. 2244/16) erlitt am 22. April 1916 eine Granatsplitterverletzung.

Der Befund ergibt verheilten Einschuß mit starker Knochendepression auf dem Körper des rechten Jochbeins mit Aussehuß rechts neben der Wirbelsäule. Die rechte Lidspalte ist deutlich enger als die linke, doch besteht kein Enophthalmus. Bei sehr enger und träge reagierender rechter Pupille ist die linke Pupille mittelweit und reagiert prompt. Während sich die linke Pupille auf Cocain ziemlich stark erweitert, zeigt, die rechte Pupille nur eine Andeutung von Erweiterung.

Bds. $E S=5 / 5$, Gesichtsfeld und Bjerrum R negativ.

Bds, $\mathrm{O}=\mathrm{n}$.

An der Spaltlampe findet sich auf dem rechten Auge beginnende feinste dunkelbraune Pigmentverstärkung in und auf dem Sphinctergebiet, dagegen $\mathrm{L}$ normaler Befund. Eine beginnende Irisatrophie ist noch nicht deutlich.

Noch deutlicher kam das in Rede stehende Bild in diesem Falle zum Ausdruck:

1) Siehe Anmerkung Seite 48 .

v. Graefes Archiv für Ophthalmologie. Bd. 97. 
Fall 27. Der 30 Jahre alte Rudolf K. (1387/17) erhielt am 3. XII. 1916 einen Streifschuß des Halses, der durch die Mitte des rechten Sternocleidomastoideus und in gleicher Höhe die Halswirbelsäule streifend genau hinten in der Medianlinie heraustretend verlaufen war. Außerdem gab er an, infolge eines Eisenbahnunglückes auch eine Quetschung der linken Halsseite erlitten zu haben. Kurz nach den beiden Unfällen sei er auf dem linken Auge erblindet und das Sehen auf dem rechten Auge immer schlechter geworden.

L S $=$ Hdb. p. d. Auge, Gl. b. n.

$\mathrm{R} \mathrm{S}=-1,5 \mathrm{D}=5 / 15$ p. Gl. b. $\mathrm{n}$.

Bd. Augen, außerlich o. B., zeigen bei der Aufnahme am 13. VII. 1917 bds. eine glaukomatöse Exkavation, $L$ mit Atrophie, $R$ ohne dieselbe.

Bei einem Druck von bds. $35 \mathrm{~mm}$ und $\mathbf{R}$ fast röhrenförmig eingeengtem Gesichtsfeld, erweitern sich bds. die Pupillen auf Cocain sehr mangelhaft, ohne daß Lidspaltenverengerung oder Enophthalmus besteht.

An der Spaltlampe findet sich beiderseits deutliche Pigmentverschiebung im leicht atrophischen Sphincter- und. Krausengebiet und die Hornhauthinterfläche ist mit Pigment bestäubt.

Am 9. XI. 1917 war der Befund noch unverändert, abgesehen von der infolge des beiderseitigen Elliots bedingten stärkeren oberflächlichen Pigmentverstreuung auf der Iris und Hornhaut.

Fall 28. Der 23 Jahre alte Emil K. (J.-N. 1483/18) erlitt im Juli 1915 durch einen Schrapnellschuß einen Einschuß am Kinn, und zwar auf der rechten Seite, während der Ausschuß sich rechtsseitig am Hinterkopf befand. Gleich nach der Verwundung wollte Patient schlecht gesehen haben, während er früher immer gut gesehen hatte. Seitdem soll auch bis jetzt das Sehen auf dem rechten Auge langsam immer schlechter geworden sein, wobei sich allmählich die Unfähigkeit herausbildete, das rechte Oberlid spontan zu heben.

Der Befund ergibt:

$\mathrm{S} R+1,0 \mathrm{D}=5 / 50$, GI. b. $\mathrm{n}$.

$\mathrm{SL}+1.0 \mathrm{D}=5 / 5$.

Das rechte Oberlid hängt herab und kann spontan nicht gehoben werden. Die Lidspalte ist um wenigstens ein Drittel rechts enger als links. Die Schleimhaut des Augapfels ist in ihrem unteren Teile mäßig gerötet, ebenso die Bindehaut des unteren Lids.

Der Bulbus selbst ist blaß, die Cornea klar, der ganze Bulbus erscheint etwas in die Augenhöhle zurïckgesunken, ist aber sonst frei beweglich.

Die rechte Pupille ist erheblich enger als die linke, sie reagiert auf Lichteinfall sowohl direkt als indirekt. Die Iris ist reizlos, der Augenhintergrund absolut normal, vielleicht sind die Venen etwas weiter als sonst. Linse und Glaskörper sind ohne Veränderung.

Die rechten Augenwimpern sind länger als die linken.

Linkes Auge äußerlich und ophthalmoskopisch absolut normal.

Der Druck beträgt $R \quad 17-20 \mathrm{~mm}$ synchron mit dem Puls, während L keine Andeutung von Puls bei $20 \mathrm{~mm}$ Druck besteht.

Das rechte Gesichtsfeld ist ungefähr konzentrisch eingeengt, namentlich auch für Rot mit besonderer Beteiligung des nasalen Sektors, so daß auch an Glaukom gedacht werden kann.

A n der Spaltla m pe links normaler Befund, dagegen findet sich rechts im Gebiete der Iriskrause und des Sphinoters sowohl als auch im Ciliarteile eine ausgedehnte dunkle Pigmentzerstäubung im Irisstroma und auf der Oberfläche. An der Hornhauthinterfläche zahlreiche Pigmentpunkte. Der Pupillarsaum in ganz leicht beginnender Depigmentierung und das ganze Irisstroma vielleicht schon in beginnender ganz leichter Rarefikation. 
Wenn auch in den geschilderten drei letzten Fällen Pigmentveränderungen der Iris, die als heterochromieähnliche hätten aufgefaßt werden können, zu vermissen waren, so ist doch das Zusammentreffen von Sympathicusläsion und glaukomatöser Pigmentverschiebung bei 2 Fällen and in einem Falle auch bei klinisch manifestem Glaukom um so bemerkenswerter, als die Möglichkeit einer Beziehung auch zwischen Heterochromie und glaukomatöser Pigmentverschiebung aus den 3 nächsten Fällen ersichtlich wurde.

Hier handelte es sich um Patienten mit typischer einseitiger Heterochromie, welche bei dem e nen Falle ohne jede Spur der bei dieser Affektion-bekannten häufigen chronischen schleichenden Iridocyclitis einherging, während $\mathrm{d}$ e beiden anderen Fälle dadurch kompliziert erschienen

Der erste Fall war dieser:

Fall 29. Bei dem 26 jahrigen Friedrich J. (J.-N. 16575/17) besteht $R$ mit $+4,0 \mathrm{D} \mathrm{S}=5 / 50, \mathrm{~L}-8,0 \mathrm{D} \mathrm{S}=$ Fgz. v. d. A., Gl. b. n. Ophth. R normaler Befund, L Fundus myopicus; die Iris zeigt typische Heterochromie gegen die leicht hellbraun pigmentierte rechte Iris.

An der Spaltlampe besteht $\mathbf{R}$ normaler Befund, dagegen $\mathbf{L}$ eine völlige Depigmentation des Pupillarsaums. Außerdem finden sich allenthalben im Sphincter- and Krausengebiete ziemlich unregelmäßig verteilt beginnende Pigmentverschiebung und zahlreiche Pigmentpunkte auf der Hornhauthinterfläche, aber noch keine deutliche stromale Irisatrophie.

Irgendwelche Zeichen einer Sympathicusläsion sind nicht feststellbar, beide Pupillen sind gleichweit, mittelgroß, rund und reagieren prompt, auch folgt auf Cocaindarreichung gute Erweiterung.

Vor allem blieb hervorzuheben, daß hier keinerlei iritische Reizung bestand und nur die Pigmentverschiebung allein nachweisbar war. Klinische Glaukomzeichen fehlten auch hier, so daß die weitere Beobachtung lehren muß, ob sich das bestehende Präglaukom zum klinisch nachweisbaren Glaukom weiterentwickeln wird. Das Fehlen jeder Zeichen einer Sympathicuserkrankung sprach nicht gegen die Auffassung, daß hier doch eine trophoneurotische Störung vorhanden war, welche sich einerseits in Form der Heterochromie, andererseits in der Pigmentversehiebung dokumentierte.

Bemerken wollen wir noch ausdrücklich, daß in 2 weiteren Fällen der Heterochromie, die im Laufe der letzten 3 Jahre hier beobachtet wurden, sich eine glaukomatöse Pigmentverschiebung an der Spaltlampe nicht nachweisen lie $\beta$, so da $\beta$ die Pigmentverschiebung an und für sich nicht $\mathrm{n}$ einem direkten und unmittelbaren Zusammenhange mit der Heterochromie zu stehen scheint.

Bei dem nächsten Falle traf die bestehende Heterochromie mit einem auch klinisch nachweisbaren Glaukom und glaukomatöser Pigmentverschiebung zusammen; ferner bestand hier nebenbei eine chro- 
nische Iridocyclitis und es war - was vor allem bemerkenswert ist auch der Augenhintergrund an der Entzündung beteiligt.

Fall 30. Die 35jährige Ida H. (181/16) war früher immer gesund, gab jedoch an, von Jugend auf ,ein helles rechtes Auge“ gehabt zu haben.

$\mathrm{L} \mathrm{E} \mathrm{S}=5 / 5 . \quad \mathrm{O}=\mathrm{n}$.

R S $=$ L. f. kl. Fl., Proj. richtig.

An der Spaltlampe sind bei leichter Ciliarreizung des Auges klümpchenförmige Hormhautbeschläge und zarte Kammerwassertrübungen sichtbar. Die Iris ist hyperämisch und zum Teil atrophisch. Am Pupillarrand sind neben einigen frischeren graulichen Knötchen viele alte Synechien vorhanden und das Pigmentepithel erscheint defekt. Die Linse ist total getrübt und in der Iris besteht schwerste Pigmentverschiebung.

$\mathrm{R}$ besteht ausgesprochene Heterochromie bei stark atrophischer Iris und ein Druck von $20 \mathrm{~mm}$ gegen $25 \mathrm{~mm}$ links.

Eine Erkrankung des Halssympathicus konnte in diesem Falle neurologisch nicht nachgewiesen werden.

Nach Extraktion der Katarakt durch Linearextraktion fanden sich $\mathrm{R}$ starke Glaskörpertrübungen und eine typische Chorioiditis und Periphlebitis, während der Befund am vorderen Bulbusabschnitte unverändert war.

Die Tuberkutinprobe sowie Wassermannreaktion waren negativ.

Nach einem halben Jahr war der Befund folgender:

$\mathrm{S}=+7,0 \mathrm{D}=5 / 15$. Druck $=25 \mathrm{~mm}$.

Die Kammerwassertrübungen und die Glaskörpertrübungen haben sich entschieden aufgehellt, doch sind noch zahlreiche Beschläge und chorioiditische Herde zu sehen neben einer deutlichen und fortgeschrittenen glaukomatösen Exkavation der Papille.

Genau das gleiche Bild bot ferner der hier längere Zeit behandelte 25jährige Student 0., doch fehlte die Exkavation (Fall 31).

Bei diesen letztgenannten beiden Fällen kam die von Heine und auch von uns in Mitteilung 10 hervorgehobene auffallende Ähnlichkeit des klinischen Bildes der Entzündung, speziell der Beschläge mit den bei der Tuberkulose zu beobachtenden Veränderungen im Spaltlampenbilde ungemein deutlich zur Wahrnehmung. Trotz negativen Verlaufes der klinischen Reaktionen bot das Spaltlampenbild sowohl bezüglich der Knötchenentwicklung am Pupillarsaum als bezüglich des Aufbaues und der Konfiguration der Beschläge die typischen Kennzeichen der Tuberkulose.

Wenn auch nach Franke, Fuchs, Lutz, Schlippe und Weill eine tuberkulöse Ätiologie bei dieser mit Heterochromie kombinierten Form der Iritis unwahrscheinlich ist, so war doch die histologische Ähnlichkeit mit Tuberkulose in vivo äußerst auffällig. Daß in dem vorliegenden Falle trotz negativer Tuberkulinprobe eine Tuberkulose dennoch als sicher anzunehmen war, ging auch aus der Mitbeteiligung des Augenhintergrundes in Form der typischen Chorioiditis und Periphlebitis hervor. Auch Frank beobachtete einen ähnlichen Fall. Dehtleffsen sah unter 12 Fällen von mit Iridocyclitis komplizierter Heterochromie nur bei 10 Fällen eine positive Tuberkulinreaktion. Vielleicht bevorzugt die Tuberkulose gerade das heterochromische Auge 
als einen von Geburt an bestehenden locus minoris resistentiae, węnn auch nicht in allen Fällen ihre Lokalisation in solchen Augen zu verfolgen ist, bezugsweise auch eine Entzündung bisher unbekannter Natur (Fuchs) sich daselbst zu etablieren scheint.

Angesichts der an der Spaltlampe nachgewiesenen gleichzeitigen glaukomatösen Pigmentversehiebung bezugsweise eines bestehenden klinischen Glaukoms - für welche bei den letzten 2 Fällen offenbleiben mußte, ob sie primärer oder sekundärer Natur waren - ist nicht zu leugnen, daß ein bisher noch nicht genügend zu definierender Zusammenhang zwischen Sympathicuserkrankung, Glaukom und Heterochromie bestehen kann. Das zeigt auch die Durchsicht der Literatur.

Auf die genauere Zusammenstellung der Literatur speziell über den Zusammenhang zwischen Sympathicuserkrankung und Glaukom ist an dieser Stelle verzichtet worden, weil das demnächst aus unserer Klinik in einer Dissertation von Fräulein M. Becker geschehen wird.

Die Beziehungen zwischen den beiden zuletzt genannten Erkrankungen dürften gerade in der glaukomatösen Pigmentverschiebung einen gewissen Ausdruck finden und auf eine gemeinsame Ursache hinweisen, die wiederum ihrerseits zur Frage eines Zusammenhanges zwischen Glaukom und Heterochromie hinüberleitet.

Auch diese Beziehung wurde bereits in der Literatur festgestellt. So erinnere ich an die Angaben von Dehtleffsen, der in 3 Fällen das Zusammentreffen von Glaukom auf dem mit Heterochromie behafteten Auge wahrnehmen konnte. Ferner hatte Mawas Gelegenheit, bei durch Heterochromie komplizierten Kataraktaugen eigentümliche degenerative Prozesse an den hinteren Epithelzellen der Iris und des Ciliarteils festzustellen, Kernzerfall und Untergang der Zellen unter eigentümlicher Vakuolisierung des Kerns, Homogenisierung des Protoplasmas und sehr unregelmäßiger Pigmentzerteilung.

Wenn somit auch eine glaukomatöse Degeneration des retinalen Irispigmentes bei der Heterochromie der Iris relativ selten vorzukommen scheint, so steht um so mehr im Vordergrunde der Prozeß des Zerfalls und der Degeneration der hellbraunen stromalen, also mesodermalen Pigmentzellen. Ob die letztere auf einer sehr frühen Pigmenternährungsstörung der Iris beruht ( $F u c h s$ ), oder ob die mangelhafte Pigmentierung als solche nur als ein Zeichen der Minderwertigkeit dieser Organe überhaupt aufzufassen ist und nicht als Krankheit oder Krankheitsursache selbst (La u ber), steht dahin. Vielleicht kann dabei überhaupt eine hellere Iris krankhaft sein im Sinne einer ersten und frühen Manifestierung eines chronisch-entzündlichen Prozesses, der mit dem Untergange der pigmentierten Stromazellen und der Bildung von Bindegewebe einhergeht.

Für die Spaltlampenuntersuchung bietet die Frage des mesodermalen 
Stromapigmentes weniger Interesse. Einerseits ist das stromale Pigment im Spaltlampenbilde überhaupt sehr flüchtig und zerfällt sehr leicht, zweitens ist sein Nachweis als Staub entschieden schwieriger als die staubartigen Zerfallsprodukte des retinalen Irispigmentes. Die Frage des mesodermalen Stromapigmentes bietet ja auch hinsichtlich der glaukomatösen Pigmentverschiebung, wie wir gesehen haben, kein spezifisches Interesse und wurde hier nur der Vollständigkeit und des Vergleiches halber nebenbei erwähnt.

Beim Überblicke des Gesagten drängt sich uns angesichts der eigentümlichen Wechselbeziehung von Heterochromie, glaukomatöser Pigmentverschiebung und Sympathicuserkrankung der Gedanke auf, daß hier nicht allein blinder Zufall walten kann, sondern ätiologische Fäden sich von dem einen zum anderen spinnen müssen. Welcher Art diese Fäden sind, ob sich z. B. die supponierte Sympathicusstörung zum Teil auf Hals- und Augensympathicussymptome oder zum Teil nur auf Augensymptome allein beschränken kann, bleibt eine offene Frage, ebenso auch das Problem, woher denn in letzter Linie die supponierte und fragliche Sympathicusstörung kommen dürfte. Ob hier Störungen der inneren Sekretion oder ähnliches eine Rolle spielen, ist ebenfalls bis auf weiteres nicht zu unterscheiden und muß weiterer diesbezüglichen Forschung vorbehalten bleiben.

Soviel dürfte jedoch nach den bisher vorliegenden Ergebnissen der Literatur sowie auch nach unseren beobachteten Fällen einigermaßen sicher erscheinen, daß wir, wenn wir zusammenfassend das ganze Problem unter einen Gesichtspunkt bringen wollen, drei Rubriken aufstellen können, die jede für sich wie auch zusammen mit den anderen je eine ätiologisch in einem gewissen Zusammenhange stehende Gruppe zu bilden vermögen und die vermutete Beziehung zwischen Sympathicusstörung, Heterochromie und Pigmentverschiebung resp. Primärglaukom zum Ausdruck bringen:

\section{Gruppe.}

Die Kombination von Sympathicusstörung mit Heterochromie: Hierher gehören die Beobachtungen von Bistis, Dehtleff sen, Galecowsky, v. Herrenschwand, Lutz, Mendel, Majou, Scalinci Seeligmüller u. a.

\section{Gruppe.}

Die.Kombination von. Sympathicusstörung mit Präglaukom resp. Glaukom:

Hierher gehören die in der genannten Dissertation angeführten Beobachtungen und unsere Fälle Nr. 26, 27 und 28.

\section{Gruppe.}

Die Kombination von Heterochromie mit Präglaukom resp. Glaukom: 
Hierher gehören die Fälle von Dehtleffsen, evtl. die Beobachtung von Mawas und unsere Fälle Nr. 29, 30 und 31.

Alle diese Gruppen sind, wie aus der Zusammenstellung hervorgeht, die Glieder eines Ringes, der in sich geschlossen erscheint und als gemeinsame Basis für alle drei Gruppen die ätiologisch noch dunkele trophoneurotische Störung im Irispigmente besitzt.

\section{Literatur.}

1. Bistis, Klin. u. experim. Unters. üb. d. Ätiol. d. Heterochrom. Archiv f. Augenheilk. 75, 4. 1913.

2. - La .paral. du sympath. dans l'étiolog. de l'hétérochr. Arch. d'Ophth. 32, 578.

3. Chotzen und Kutznitzky, Die Strahlenbeh. d. A. I. Mittlg. Klin. Mon. f. A. 60, 1918.

4. Dehtleffsen, 77 Fälle von Heterochr. irid. Inaug.-Diss. Kiel 1912.

5. Elschnig, Kriegsver. d. A. Mediz. Klinik 20. 1915.

6. Franke, F., Heterochr. d. Regenbogenh. u. Augenerkr. Klin. Monatsbl. f. Augenheilk. 58, 165.1917.

7. Fuchs, Über endogen. Uveit. usw. Archiv f. Ophthalmol. 38.

8. - Über Kompl. b. d. Heterochr. Zeitschr. f. Augenheilk. 15, 191. 1906.

9. - Über Heterochr. usw. Archiv f. Ophthalmol 93, 4. 1917.

10. Galecowsky, Heterochr. de l'Iris, catar. et troubles sympath. Bull. de la Soc. d'Ophth. de Paris. März 1911.

11. - D. Verfärb. d. Regenbogenh. Rec. d'Ophth. 1910 S. 101.

12. - Rec. d'Ophth. 43. 1912.

13. Hei ne, Erf. u. Ged, über Tuberk. u. Tub. Med. Klin. 44 u. 45. 1912.

14. Hertel, zit. n. (15).

15. v. Herrenschwand, F., Ubb. versch. Art. v. Heter. iridis. Klin. Mon. f. A. 60. 1918.

16. Höhmann, Über d. Pigments. d. Pupillarr., seine individ. Versch. usw. Archiv f. Augenheilk. 60, 60. 1912.

17. Jickeli, C., Einseit. Sympathicusl. n. Sehußverl. Klin. Monatsbl. f. Augenheilk. 56, 538. 1916.

18. Koeppe, L., Die Rolle d. Irispigm. b. Glaukom. Heidelberger Bericht 1916.

19. - Klin. Beob. m. d. Nernstsp. usw. Mitteilung 3. v. Graefes Archiv f. Ophthalmol. 92, 3. 1916; ferner Mitteilung 12. Ebenda; Mitteilung 10.

20. de Lapersonne, Syndr. oculo-sympt. etc. Arch. d'Ophth. 34, 9. 1915.

21. La uber, Anat. Unters. über Heterochr. b. tauben, unvollk, albin. Katzen. Zeitschr. f. Augenheilk. 16, 326. 1916 .

22. Liebrecht, Gesichtsschuß. Deutsche Med. Wochenschr. 1915, S. 514.

23. Lutz, Über einige Fälle v. Heterochr. irid. Zeitschr. f. Augenheilk. 19. 1908.

24. Majou, Paral. of the sympath. with slight heterochr. iridis. Ophthalmoskope August 1916.

25. - Two cases of the symp. Ophthalmoskope Juni 1916.

26. - Transact of of the ophth. soc. Vol. 29, 3. 1909.

27. Mawas, Contrib. à l'étude du rapp. etc. Trav. de la Fondat. A. de Rothsohild 1911, S. 119.

28. Mende 1, zit. n. (15).

29. Metzner u. Wölfflin, Klin. u. experim. Unters. über Halssympathiousl. Archiv f. Ophthalmol. 89, 2. 1915. 
56 L. Koeppe: Klinische Beobachtungen mit der Nernstspaltlampe usw. XiII.

30. Metzner u. Wölfflin, Ebenda 91, 2. 1916.

31. Mohr, zit. n. (15).

32. Reinhardt, zit. n. (5).

33. Roche, Les paralys. du symp. cervic. dans les bless. de guerre. Arch. d'Ophth. November/Dezember 1916.

34. Russel1, 2 Fälle von Lähmung d. Halssympath. m. Augenersch. b. Kriegsverl. Zeitschr. f. Augenheilk. 33, 5/6. 1916.

35 Saenger, zit. n. (5).

36. Scalinci, N., Eterocromia e paralisi del simpatico. Aroh. di Ottalmol. Vol. 22, 2.

37. Schlippe, Über d. klin. u. anat. Irisbefund usw. Archiv f: Augenbeilk. 67, 2 u. 3.

38. Seeligmüller, zit. n. (15).

39. Uhth off, Z. Kataraktoper. b. Diabetikern. Heidelberger Berichte 1908.

40. Weill, G., Über Heterophthalm. Zeitschr. f. Augenheilk. 11, 165.

41. Wessely, Augenärztl. Erfahr. i. Felde. Würzburger Abhandlung aus d. Gesamtg. d. Med. 15, 9. 1915. 
v. Graefes Archiv Bd. 97.

Tafel II.

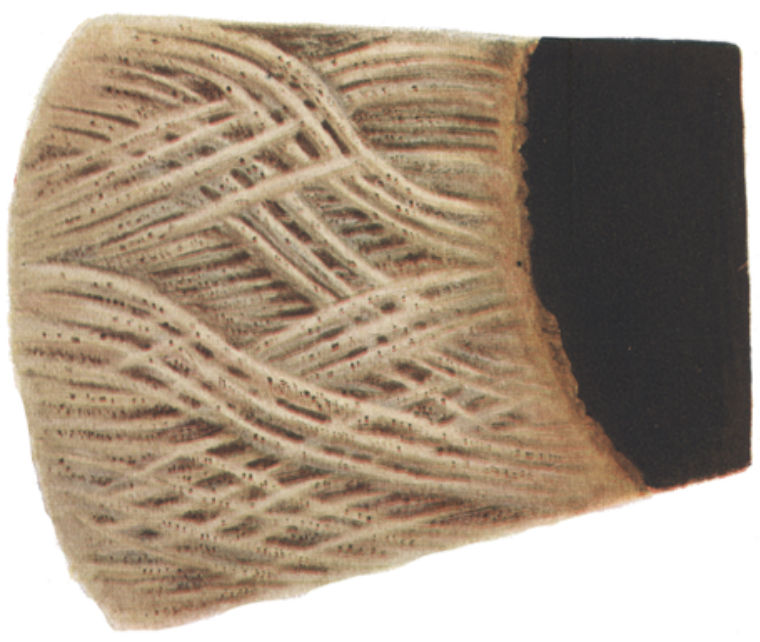

Sphincterstück der Iris bei Glaucom.

K. Wangerin pinx.

Ko e p p. Klin. Beobachtungen mit der Nernstspaltlampe XII. Verlag von Julius Springer in Berlin. 